\title{
Application of the Fröbenius Method to the Schrödinger Equation for a Spherically Symmetric Hyperbolic Potential
}

\author{
Hippolyte Nyengeri¹, Rénovat Nizigiyima', Eugène Ndenzako', Félix Bigirimana², \\ Dieudonné Niyonkuru ${ }^{3}$, Audace Girukwishaka ${ }^{1}$ \\ ${ }^{1}$ Department of Physics, Faculty of Science, University of Burundi, Bujumbura, Burundi \\ ${ }^{2}$ Petit Séminaire de Mika, Rutana, Burundi \\ ${ }^{3}$ Faculty of Science and Technology, East African Leadership University, Muyinga, Burundi \\ Email: hippolyte.nyengeri@ub.edu.bi
}

How to cite this paper: Nyengeri, H., Nizigiyima, R., Ndenzako, E., Bigirimana, F., Niyonkuru, D. and Girukwishaka, A. (2018) Application of the Fröbenius Method to the Schrödinger Equation for a Spherically Symmetric Hyperbolic Potential. Open Access Library Journal, 5: e4950. https://doi.org/10.4236/oalib.1104950

Received: September 29, 2018

Accepted: October 26, 2018

Published: October 29, 2018

Copyright $\odot 2018$ by authors and Open Access Library Inc.

This work is licensed under the Creative Commons Attribution International License (CC BY 4.0).

http://creativecommons.org/licenses/by/4.0/

\begin{abstract}
In this paper, an efficient technique for computing the bound state energies and wave functions of the Schrödinger Equation (SE) associated with a new class of spherically symmetric hyperbolic potentials is developed. This technique is based on a recent approximation scheme for the orbital centrifugal term and on the use of the Fröbenius method (FM). The bound state eigenvalues are given as zeros of calculable functions. The corresponding eigenfunctions can be obtained by substituting the calculated energies into the recurrence relations for the expanding coefficients of the Fröbenius series representing the solution. The excellent performance of this technique is illustrated through numerical results for some special cases like Pöschl-Teller potential (PTP), Manning-Rosen potential (MRP) and Pöschl-Teller polynomial potential (PTPP), with an application to the Gaussian potential well $(\mathrm{GPW})$. Comparison with other methods is presented. Our results agree noticeably with the previously reported ones.
\end{abstract}

\section{Subject Areas \\ Quantum Mechanics}

\section{Keywords}

Fröbenius Method, Schrödinger Equation, Hyperbolic Potential, Bound States, Wave Functions

\section{Introduction}

It is well known that the exact solution of the Schrödinger Equation (SE) plays a 
vital role in quantum mechanics, and solving this equation is still an interesting work in the existing literature [1]-[6]. Generally, the SE is very difficult to solve for most physical central potentials [7] [8] [9]. It is for this reason that approximation and numerical methods are frequently used to arrive at the solution [6] [10]-[15].

Recently, the study of hyperbolic and exponential-type potentials has attracted a lot of interest by many authors due to their wide range of applications in physics [1] [2] [4]-[10] [14] [16]-[21]. However, the bound state solutions can only be obtained analytically for some of these potentials among which Pöschl-Teller [3] [9] [16] [17], Hulthen [8], Manning-Rosen [1] [2] [7] [8] and Rosen-Morse [3] [16] [18] [21] potentials. Moreover, when, in three-dimensional space, the angular momentum quantum number is non zero, one can only solve the SE approximately using a suitable approximation scheme for the centrifugal term [3]. Such an approximation scheme includes the conventional approximation scheme proposed by Greene and Aldrich [13] [22]; the improved approximation scheme [18] [22] [23] suggested by Jia, Chen and Cui; the elegant approximation scheme [24] [25]; the approximation scheme proposed by Wei and Dong [25] and a recent approximation scheme suggested by Chen, Lou and You , and given by [9]:

$$
\frac{1}{r^{2}} \approx \varphi^{2}\left[\frac{\&}{\sinh ^{2}(\varphi r)}+\frac{t}{\cosh ^{2}(\varphi r)}\right]
$$

where $\varphi, \&$ and $t$ are adjustable dimensionless parameters.

In this work, we use the approximation scheme (1) to deal with the centrifugal term and solve, by means of the Fröbenius method (FM), the SE with the class of hyperbolic potentials defined by

$$
\begin{aligned}
V(r)= & C_{-2} \operatorname{coth}^{2}(\alpha r)+C_{-1} \operatorname{coth}(\alpha r)+\sum_{i=1}^{3} C_{i} \tanh ^{i}(\alpha r) \\
& +\sum_{j=1}^{K} B_{j} \operatorname{sech}^{2 j}(\alpha r)
\end{aligned}
$$

where $C_{-2}, C_{-1}, C_{1}, C_{2}, C_{3}, B_{1}, \cdots, B_{K} \in \mathbb{R} ; \alpha \in \mathbb{R}_{0}$ and $K \in \mathbb{N}_{0}$. This class includes many potentials, among which:

- The modified Pöschl-Teller potential (PTP) [26] which has been used to describe bending molecular vibrations [27] [28] [29];

- The PTP of the form [17]

$$
V_{P T}(r)=\frac{-A(A+\alpha)}{\cosh ^{2}(\alpha r)}+\frac{B(B-\alpha)}{\sinh ^{2}(\alpha r)}
$$

This potential is an important diatomic molecular potential which has wide applications in physics and chemical physics [30]. It has been used to account for the physics of many systems which includes the excitons, quantum wires, and quantum dots [31];

- The Rosen-Morse potential (RMP) [18] [21] and the Manning potential [32] which are generally used to explain vibrations of polyatomic molecular 
structures [21] [32] [33] [34] [35];

- The Manning-Rosen potential (MRP) [8] [36] which is an important mathematical model for molecular vibrations and rotations [37] [38];

- The Hartmann potential [20]. This potential exhibits in general an asymmetric double-well. It is therefore hoped that it can serve as a useful tool in the study of phenomena whose behavior is described by asymmetric double-wells [20].

We have to add that the class of potentials under consideration contains all the approximation schemes of the orbital centrifugal term mentioned above. It also can be used to model the Gaussian potential well (GPW) through the expression [6]

$$
-\exp \left(-r^{2}\right) \approx \sum_{j=1}^{8} \mathcal{B}_{j} \operatorname{sech}^{2 j}(\alpha r)
$$

where $\alpha, B_{1}, \cdots, B_{8}$ are adjustable parameters.

Based on the foregoing, it can be said that the potential under consideration can be reduced to a lot of potentials which are known to play a very important role in many fields of physics such as molecular physics, solid state, and chemical physics. Consequently, it is for considerable interest to find general solution of its associated SE.

The rest of this paper is organized as follows. In Section 2, we apply the approximation (1) to the exact $\mathrm{SE}$, and then convert the resulting equation into a dimensionless eigenvalue problem that can be solved by the use of the FM. In Section 3, we establish the Fröbenius series solution of this problem and show how to determine the bound state spectrum. Section 4 is devoted to three special cases, namely, the MRP, the PTP and the Pöschl-Teller polynomial potential (PTPP), with an application to the GPW in three dimensional space. In each case, the bound state energies obtained by means of the FM are presented and compared with those given by other methods. Finally, we present our conclusions in Section 5.

\section{The Approximate Radial Schrödinger Equation}

The time-independent SE for a particle of mass $M$ that moves in three-dimensional space under the effect of a spherically symmetric potential is given by

$$
-\frac{\hbar^{2}}{2 M}\left[\Delta_{r}+V(r)\right] \Psi(\vec{r})=E \Psi(\vec{r})
$$

Taking $\Psi(\vec{r})=r^{-1} \psi(r) Y_{\ell m}(\theta, \varphi)$ and considering potential (2), we obtain the radial $\mathrm{SE}$ as

$$
\begin{aligned}
& -\frac{\hbar^{2}}{2 M} \frac{\mathrm{d}^{2} \psi(r)}{\mathrm{d} r^{2}}+\left[\frac{\ell(\ell+1)}{2 M r^{2}}+C_{-2} \operatorname{coth}^{2}(\alpha r)+C_{-1} \operatorname{coth}(\alpha r)\right. \\
& \left.+\sum_{i=1}^{3} C_{i} \tanh ^{i}(\alpha r)+\sum_{j=1}^{K} B_{j} \operatorname{sech}^{2 j}(\alpha r)\right] \psi(r)=E \psi(r)
\end{aligned}
$$

Using a new variable of the form

$$
R=b r, b \in] 1,+\infty[
$$


we obtain the following differential equation:

$$
\begin{aligned}
& -\frac{\hbar^{2} b^{2}}{2 M} \frac{\mathrm{d}^{2} \psi(R)}{\mathrm{d} R^{2}}+\left[\frac{\ell(\ell+1) b^{2}}{2 M R^{2}}+C_{-2} \operatorname{coth}^{2}(\tilde{\alpha} R)+C_{-1} \operatorname{coth}(\tilde{\alpha} R)\right. \\
& \left.+\sum_{i=1}^{3} C_{i} \tanh ^{i}(\tilde{\alpha} R)+\sum_{j=1}^{K} B_{j} \operatorname{sech}^{2 j}(\tilde{\alpha} R)\right] \psi(R)=E \psi(R)
\end{aligned}
$$

where $\tilde{\alpha}=\alpha / b$. Obviously, Equation (8) cannot be solved exactly when $\ell \neq 0$ due to the centrifugal term $1 / R^{2}$. To reduce this equation into a differential equation that can be solved by means of the FM, we must use a suitable approximation for the centrifugal term. We here consider the approximation scheme (1) proposed by Chen, Lu and You [9]. Substituting this approximation into Equation (8) and making the change of variable $\xi=\tanh (\tilde{\alpha} R)$, such that the domain $0<r<\infty$ maps to $0<\xi<1$, we find

$$
\begin{aligned}
& \xi^{2}\left(\xi^{2}-1\right)^{2} \frac{\mathrm{d}^{2} \psi(\xi)}{\mathrm{d} \xi^{2}}+2 \xi^{2}\left(\xi^{2}-1\right) \frac{\mathrm{d} \psi(\xi)}{\mathrm{d} \xi} \\
& +\frac{2 M}{\hbar^{2} \alpha^{2}}\left[-\left(C_{-2}+\frac{\ell(\ell+1) \alpha^{2} \diamond}{2 M}\right)-C_{-1} \xi+\left(E+\frac{\left.\ell(\ell+1) \alpha^{2} \diamond\right)}{2 M}\right) \xi^{2}\right. \\
& \left.-C_{1} \xi^{3}-C_{2} \xi^{4}-C_{3} \xi^{5}-\sum_{j=1}^{K} \tilde{B}_{j}\left(1-\xi^{2}\right)^{j} \xi^{2}\right] \psi(\xi)=0
\end{aligned}
$$

where the $\tilde{B}_{j}$ coefficients are such that

$$
\tilde{B}_{1}=\frac{\ell(\ell+1) \alpha^{2} t}{2 M}+B_{1} ; \quad \tilde{B}_{j}=B_{j}, \quad j=2,3, \cdots, K
$$

\section{Expansion around a Regular Singular Point}

It is clear that $\xi=1$ and $\xi=0$ are both regular singular points for the differential Equation (9). The FM can therefore be applied with the wave function represented as generalized power series

$$
\psi(\xi)=\xi^{\delta} \sum_{n=0}^{\infty} a_{n} \xi^{n}
$$

or

$$
\psi(\xi)=(\xi-1)^{\delta} \sum_{n=0}^{\infty} a_{n}(\xi-1)^{n}
$$

where $a_{0} \neq 0$. Let us seek $\psi(\xi)$ in the form (12). We first make the substitution $\eta=\xi-1$. The resulting differential equation can be written as

$$
\begin{aligned}
& \left(4 \eta^{2}+12 \eta^{3}+13 \eta^{4}+6 \eta^{5}+\eta^{6}\right) \frac{\mathrm{d}^{2} \psi(\eta)}{\mathrm{d} \eta^{2}}+\left(4 \eta+14 \eta^{2}+18 \eta^{3}+10 \eta^{4}+2 \eta^{5}\right) \frac{\mathrm{d} \psi(\eta)}{\mathrm{d} \eta} \\
& +\frac{2 M}{\hbar^{2} \alpha^{2}}\left[-\left(C_{-2}+\frac{\ell(\ell+1) \alpha^{2} \text { ॰ }}{2 M}\right)-C_{-1}(1+\eta)+\left(E+\frac{\ell(\ell+1) \alpha^{2} \text { ॰ }}{2 M}\right)\left(1+2 \eta+\eta^{2}\right)\right. \\
& \left.-C_{1}(1+\eta)^{3}-C_{2}(1+\eta)^{4}-C_{3}(1+\eta)^{5}-\sum_{j=1}^{K} \hat{B}_{j} \eta^{j}(1+\eta)^{2}\right] \psi(\eta)=0
\end{aligned}
$$


where the $\hat{B}_{j}$ coefficients are calculated from the two following equations:

$$
\begin{aligned}
& \hat{B}_{2 p+1}= \sum_{\substack{j=p+1 \\
j \leq K}}^{2 p+1}(-1)^{j}\left(\begin{array}{c}
j \\
2 p+1-j
\end{array}\right) 2^{2 j-2 p-1} \tilde{B}_{j} ; p=0,1, \cdots, K-1 \\
& \hat{B}_{2 p}=\sum_{\substack{j=p \\
j \leq K}}^{2 p}(-1)^{j}\left(\begin{array}{c}
j \\
2 p-j
\end{array}\right) 2^{2 j-2 p} \tilde{B}_{j} ; p=1,2, \cdots, K
\end{aligned}
$$

It can be verified that

$$
\sum_{p=1}^{2 K} \hat{B}_{p} \eta^{p}(1+\eta)^{2}=\sum_{p=1}^{2 K+2} \beta_{p} \eta^{p}
$$

where

$$
\begin{gathered}
\beta_{1}=\hat{B}_{1} ; \quad \beta_{2}=2 \hat{B}_{1}+\hat{B}_{2} ; \quad \beta_{j}=\hat{B}_{j-2}+2 \hat{B}_{j-1}+\hat{B}_{j}, j=3,4, \cdots, 2 K \\
\beta_{2 K+1}=\hat{B}_{2 K-1}+2 \hat{B}_{2 K} ; \quad \beta_{2 K+2}=\hat{B}_{2 K} .
\end{gathered}
$$

Consequently, Equation (13) can be rewritten as

$$
\begin{aligned}
& \left(4 \eta^{2}+12 \eta^{3}+13 \eta^{4}+6 \eta^{5}+\eta^{6}\right) \frac{\mathrm{d}^{2} \psi(\eta)}{\mathrm{d} \eta^{2}} \\
& +\left(4 \eta+14 \eta^{2}+18 \eta^{3}+10 \eta^{4}+2 \eta^{5}\right) \frac{\mathrm{d} \psi(\eta)}{\mathrm{d} \eta} \\
& +\left[d_{0}+\varepsilon+\left(d_{1}+2 \varepsilon\right) \eta+\left(d_{2}+\varepsilon\right) \eta^{2}+\sum_{i=3}^{5} d_{i} \eta^{i}+\sum_{p=6}^{2 K+2} b_{p} \eta^{p}\right] \psi(\eta)=0
\end{aligned}
$$

where

$$
\begin{aligned}
& \varepsilon=\frac{2 M}{\hbar^{2} \alpha^{2}} E ; \quad d_{0}=-\frac{2 M}{\hbar^{2} \alpha^{2}}\left[C_{-2}+C_{-1}+C_{1}+C_{2}+C_{3}\right] \\
& d_{1}=\frac{2 M}{\hbar^{2} \alpha^{2}}\left[\frac{2 \ell(\ell+1) \alpha^{2} \triangleleft}{2 M}-C_{-1}-3 C_{1}-4 C_{2}-5 C_{3}-\beta_{1}\right] \\
& d_{2}=\frac{2 M}{\hbar^{2} \alpha^{2}}\left[\frac{\ell(\ell+1) \alpha^{2} \vartheta}{2 M}-3 C_{1}-6 C_{2}-10 C_{3}-\beta_{2}\right] \\
& d_{3}=-\frac{2 M}{\hbar^{2} \alpha^{2}}\left[C_{1}+4 C_{2}+10 C_{3}+\beta_{3}\right] ; \quad d_{4}=-\frac{2 M}{\hbar^{2} \alpha^{2}}\left[C_{2}+5 C_{3}+\beta_{4}\right] \\
& d_{5}=-\frac{2 M}{\hbar^{2} \alpha^{2}}\left(C_{3}+\beta_{5}\right) ; \quad b_{j}=-\frac{2 M}{\hbar^{2} \alpha^{2}} \beta_{j}, \quad j=6,7, \cdots, 2 K+2
\end{aligned}
$$

Note that Equation (19) can be solved near $\eta=0$ by writing

$$
\psi(\eta)=\eta^{\delta} \sum_{n=0}^{\infty} a_{n} \eta^{n}
$$

Substitution of Equation (25) into (19) leads to the recurrence relation

$$
\begin{aligned}
& {\left[4+d_{0}+\varepsilon+4 \delta(2+\delta)+8 n(1+\delta)+4 n^{2}\right] a_{n+1}+\left[d_{1}+2 \varepsilon+2 \delta(1+6 \delta)\right.} \\
& \left.+2 n(1+12 \delta)+12 n^{2}\right] a_{n}+\left[8+d_{2}+\varepsilon-(21-13 \delta) \delta-(21-26 \delta) n\right. \\
& \left.+13 n^{2}\right] a_{n-1}+\left[16+d_{3}-2 \delta(10-3 \delta)-4 n(5-3 \delta)+6 n^{2}\right] a_{n-2} \\
& +\left[6+d_{4}-(5-\delta) \delta-(5-2 \delta) n+n^{2}\right] a_{n-3}+d_{5} a_{n-4}+\sum_{p=6}^{2 K+2} b_{p} a_{n-p+1}=0
\end{aligned}
$$


for the expanding coefficients, with the understanding that $a_{i}=0$ for $i<0$. Setting $n=-1$ in the above relation, we obtain the indicial equation $\left(4 \delta^{2}+d_{0}+\varepsilon\right) a_{0}=0$ which is solved by $\delta_{1}=-\frac{1}{2} \sqrt{-d_{0}-\varepsilon}$ and $\delta_{2}=\frac{1}{2} \sqrt{-a_{0}-\varepsilon}$.

We can therefore conclude that the two solutions of the radial SE obtained as generalized series, one with $\delta=\delta_{1}$ and the other with $\delta=\delta_{2}$, are linearly independent. The value of $\delta$ determines the behavior of $\psi(\eta)$ for $\eta \rightarrow-1$ $(r \rightarrow 0)$ and $\eta \rightarrow 0 \quad(r \rightarrow \infty)$. Clearly, only $\delta>0$ is acceptable, since only in this case $\psi(r=0)=0$. Such a solution to the radial SE contains only the series with $\delta=\delta_{2}$, and in the following will be denoted by

$$
\psi(\eta, \varepsilon)=\eta^{\delta_{2}} \sum_{n=0}^{\infty} a_{n} \eta^{n}
$$

where the dependence on the energy eigenvalue is explicitly marked. The function $\psi(\eta, \varepsilon)$ has an important property that $\psi(\eta=0, \varepsilon)=0$, which can be regarded as a boundary condition for the radial equation at $r=\infty$. If $\psi(\eta, \varepsilon)$ is associated with a bound state, it has another important property, i.e., $\psi(\eta=-1, \varepsilon)=0$, which can be considered as the boundary condition for bound state wave function at $r=0$. It means that for arbitrary $\varepsilon$, the wave function $\psi(r, \varepsilon)$ obtained from (27) is not square integrable. If, for a particular value of $\varepsilon, \psi(\eta=-1, \varepsilon)=0$, the wave function is square integrable. In other words, this condition determines the energies of the discrete spectrum. We have to emphasize that in practice, the function $\psi(\eta, \varepsilon)$ has to be approximated by truncating the series in Equation (27) at suitably high order $N$, which requires high precision computing since some of the expanding coefficients $a_{i}$ in Equation (27) are extremely big for high values of $i$, especially when the $C_{i}$ and/or the $B_{j}$ parameters are significantly large, and $a_{0}$ is not sufficiently small. To deal with this problem, one can appeal to a new software package for arbitrary precision computation, named MPFUN 2015 and developed by David H. Bailey [39]. In our calculations, the precision level is set to 60 digits and $a_{0}$ equals $10^{-9}$. The number of terms in the series (27) is 501 . We have chosen fortran 90 as the programming language. One has to add that the truncated series is a function of $\varepsilon$ whose zeros correspond to bound state values of $\varepsilon$.

Before finishing this section, we have to indicate that when the class of potentials (2) is subject to the condition that $C_{-1}=C_{1}=C_{3}=0$, we found it most appropriate to make the change of variable $\xi=\operatorname{sech}^{2}(\tilde{\alpha} R)$ instead of using the transformation $\xi=\tanh (\tilde{\alpha} R)$. The radial SE can in this case be modified as

$$
\begin{aligned}
& \xi^{2}(1-\xi) \frac{\mathrm{d}^{2} \psi(\xi)}{\mathrm{d} \xi^{2}}+\xi\left(1-\frac{3}{2} \xi\right) \frac{\mathrm{d} \psi(\xi)}{\mathrm{d} \xi} \\
& +\frac{1}{4}\left\{\varepsilon-\left[c_{-2}+\frac{\alpha^{2}}{\hbar^{2}} \ell(\ell+1) \text { ə }\right] \frac{1}{1-\xi}\right. \\
& \left.-\left[c_{2}-\ell(\ell+1) \frac{\alpha^{2}}{\hbar^{2}} \text { ə] }\right]-\sum_{j=1}^{K} \tilde{b}_{j} \xi^{j}\right\} \psi(\xi)=0
\end{aligned}
$$


where

$$
\varepsilon=\frac{2 M}{\hbar^{2}} E, \quad c_{-2}=\frac{2 M}{\hbar^{2}} C_{-2}, \quad c_{2}=\frac{2 M}{\hbar^{2}} C_{2}, \quad \tilde{b}_{j}=\frac{2 M}{\hbar^{2}} \tilde{B}_{j}
$$

By putting the series (12) and its two first derivatives into Equation (28), and then changing variables to $\eta=\xi-1$, we obtain the recurrence relation

$$
\begin{aligned}
& {\left[(n+\delta+1)\left(n+\delta+\frac{1}{2}\right)-\frac{c_{-2}}{4}-\frac{\alpha^{2}}{4 \hbar^{2}} \ell(\ell+1) \text { ॰ }\right] a_{n+1}} \\
& +\left[2(n+\delta)^{2}-\frac{\varepsilon-c_{2}-b_{0}}{4}-\frac{\alpha^{2}}{4 \hbar^{2}} \ell(\ell+1) \text { ə }\right] a_{n} \\
& +\left[(n+\delta-1)\left(n+\delta-\frac{1}{2}\right)+\frac{b_{1}}{4}\right] a_{n-1}+\frac{b_{2}}{4} a_{n-2}+\cdots+\frac{b_{K}}{4} a_{n-K}=0
\end{aligned}
$$

for the expanding coefficients. The $b_{j}$ coefficients are such that

$$
b_{0}=\sum_{j=1}^{K} \tilde{b}_{j} ; \quad b_{j}=\sum_{i=j}^{K}\left(\begin{array}{l}
i \\
j
\end{array}\right) \tilde{b}_{i}, \quad j=1,2, \cdots, K
$$

The indicial equation, namely $\delta^{2}-\frac{1}{2} \delta-\frac{a_{-2}}{4}-\frac{\alpha^{2}}{4 \hbar^{2}} \ell(\ell+1) \curvearrowright=0$ has two solutions, i.e.,

$$
\delta_{1}=\frac{1 / 2-\sqrt{1 / 4+c_{-2}-\frac{\alpha^{2}}{\hbar^{2}} \ell(\ell+1)}}{2}, \delta_{2}=\frac{1 / 2+\sqrt{1 / 4+c_{-2}-\frac{\alpha^{2}}{\hbar^{2}} \ell(\ell+1)}}{2}
$$

When $\delta>0$, the boundary conditions for bound wave functions are satisfied.

\section{Numerical Results for Some Special Cases}

To test the accuracy of our approximate results, we consider three special cases, namely the case of the MRP [1] [2], that of the Second Pöschl-Teller potential (SPTP) [40] and the case where $V(r)=\sum_{j=1}^{K} B_{j} \operatorname{sech}^{2}(\alpha r)$, with an application to the Gaussian potential well (GPW) [41] [42]. For each case, we give a numerical comparison of the energy eigenvalues obtained by using the FM with those obtained with the use of other methods.

\subsection{The Manning-Rosen Potential}

The MRP is defined by [1] [2] [7] [8]:

$$
V(r)=\frac{\hbar^{2}}{2 M b^{2}}\left[\frac{a(a-1)}{\left(\mathrm{e}^{r / b}-1\right)^{2}}-\frac{A}{\mathrm{e}^{r / b}-1}\right]=\frac{\hbar^{2}}{2 M b^{2}}\left[\frac{a(a-1) \mathrm{e}^{-2 r / b}}{\left(1-\mathrm{e}^{-r / b}\right)^{2}}-\frac{A \mathrm{e}^{-r / b}}{1-\mathrm{e}^{-r / b}}\right]
$$

Here, a and the strength parameter $A$ are two dimensionless parameters, while the screening parameter $b$, having a dimension of length, is related to the range of potential. It can be verified that the class of potentials (2) reduces to MRP by choosing 


$$
\begin{gathered}
K=1, \quad C_{-2}=a(a-1) \hbar^{2} /\left(8 M b^{2}\right), \quad C_{-1}=-[a(a-1)+A] \hbar^{2} /\left(4 M b^{2}\right), \\
C_{1}=0, C_{2}=B_{1}=[a(a-1)+2 A] \hbar^{2} /\left(8 M b^{2}\right) \text { and } \alpha=1 /(2 b) .
\end{gathered}
$$

The MRP is used as an important mathematical model for molecular vibrations and rotations [37] [38]. It has considerable applications in several bound state and scattering problems in physics. For $a=0$ or 1 , this potential reduces to the familiar short-range Hulthen potential [8] having useful applications in nuclear, particle, solid-state and chemical physics. There is a relative minimum value at $r_{0}=b \ln [1+2 a(a-1) / A]$ with value $V\left(r_{0}\right)=-\hbar^{2} A^{2} /\left[8 M b^{2} a(a-1)\right]$ for $a<0$ or $a>1$ and $A>0$.

Let us indicate that approximate analytical solutions of $\ell$-wave SE for the MRP have been presented in [2] by a proper approximation of the centrifugal term. Furthermore, a purely numerical integrating procedure has been programmed [43] as well for bound states, invoking the Mathematica package, which offers decent results, especially in the short potential well, i.e., small $\ell$ and $a$.

In Table 1 , we report our calculated energies for selected $\ell \neq 0$ states having principal quantum number $n \leq 6$, two different values of parameter $a$, $A=2 b$ and three different values of the screening parameter $b$. The parameter $b$ has been chosen such that $\tilde{\alpha}=0.0001$, which implies that $s=0.9999999999999999864889$ and $t=0.3333422254140179276$. Our results are compared to both the previous ones [2] and the exact results obtained with the aid of the Mathematica package program by Lucha and Schöberl [43] as shown in Table 1. It is obvious that the present results are in good agreement with the accurate numerical ones and those calculated by Qiang and Dong [2].

\subsection{The Second Pöschl-Teller Potential (SPTP)}

We here consider the PTP given by [40]

$$
V(r)=-\frac{\hbar^{2} \alpha^{2}}{2 M}\left[\frac{\lambda(\lambda+1)}{\cosh ^{2}(\alpha r)}-\frac{\kappa(\kappa-1)}{\sinh ^{2}(\alpha r)}\right]
$$

where the parameters $\lambda$ and $\kappa$ are relevant to the potential depth, while $\alpha$ describes the width of the potential. The SPTP can be obtained from the class of potentials (2) by choosing $K=1$ and

$$
C_{-2}=\frac{\hbar^{2} \alpha^{2}}{2 M} \kappa(\kappa-1)=-C_{2}, \quad C_{-1}=C_{1}=0, B_{1}=-\frac{\hbar^{2} \alpha^{2}}{2 M}[\lambda(\lambda+1)+\kappa(\kappa-1)]
$$

We show, in Table 2, the energy levels calculated by the FM, those obtained numerically by means of the Mathematica package developed by Lucha and Schöberl [43] and the approximate analytical ones [40], respectively for a number of given principal quantum number $n$ and angular quantum number $\ell$ with some typical values of parameter $\alpha$. It is found that our results agree noticeably with numerically exact ones. Some of them are even better than those obtained by Chen and Dong [40]. 
Table 1. Eigenvalues as a function of $b$ for $2 \mathrm{p}-6 \mathrm{~g}$ states and for $a=0.75$ and $a=1.5$; $A=2 b, \quad s=0.9999999999999999864889$ and $t=0.3333422254140179276$.

\begin{tabular}{|c|c|c|c|c|c|c|c|}
\hline \multirow{2}{*}{ States } & \multirow{2}{*}{$1 / \mathrm{b}$} & \multicolumn{3}{|c|}{$\alpha=0.075$} & \multicolumn{3}{|c|}{$\alpha=1.50$} \\
\hline & & Present & Previous [2] & Lucha et al. & Present & Previous [2] & Lucha et al. \\
\hline \multirow[t]{3}{*}{$2 p$} & 0.025 & -0.1205309 & -0.1205279 & -0.1205271 & -0.0899711 & -0.0899721 & -0.0899708 \\
\hline & 0.050 & -0.1082184 & -0.1082232 & -0.1082151 & -0.0800443 & -0.0800492 & -0.0800400 \\
\hline & 0.075 & -0.0964609 & -0.0964683 & -0.0964469 & -0.0705910 & -0.0705918 & -0.0705701 \\
\hline \multirow[t]{3}{*}{$3 p$} & 0.025 & -0.0458801 & -0.0458801 & -0.0458779 & -0.0369148 & -0.0369157 & -0.0369134 \\
\hline & 0.050 & -0.0350796 & -0.0350717 & -0.0350633 & -0.0272909 & -0.0272769 & -0.0272696 \\
\hline & 0.075 & -0.0256403 & -0.0255735 & -0.0255654 & -0.0190415 & -0.0189482 & -0.0189474 \\
\hline \multirow[t]{3}{*}{$3 d$} & 0.025 & -0.0447766 & -0.0447810 & -0.0447743 & -0.0394818 & -0.0394860 & -0.0394789 \\
\hline & 0.050 & -0.0337291 & -0.0337217 & -0.0336930 & -0.0294922 & -0.0294773 & -0.0294496 \\
\hline & 0.075 & -0.0239358 & -0.0238044 & -0.0237621 & -0.0206654 & -0.0205009 & -0.0204663 \\
\hline \multirow[t]{3}{*}{$4 p$} & 0.025 & -0.0208136 & -0.0208119 & -0.0208097 & -0.0171783 & -0.0171761 & -0.0171740 \\
\hline & 0.050 & -0.0117829 & -0.0117351 & -0.0117365 & -0.0089680 & -0.0089080 & -0.0089134 \\
\hline & 0.075 & -0.0052697 & -0.0050420 & -0.0050945 & -0.0033786 & -0.0031128 & -0.0031884 \\
\hline \multirow[t]{3}{*}{$4 d$} & 0.025 & -0.0203108 & -0.0203087 & -0.0203017 & -0.0182218 & -0.0182182 & -0.0182115 \\
\hline & 0.050 & -0.0111163 & -0.0109919 & -0.0109904 & -0.0096551 & -0.0095129 & -0.0095167 \\
\hline & 0.075 & -0.0045354 & -0.0038988 & -0.0040331 & -0.0036691 & -0.0029754 & -0.0031399 \\
\hline \multirow[t]{3}{*}{$4 f$} & 0.025 & -0.0199931 & -0.0199948 & -0.0199797 & -0.0186284 & -0.0186287 & -0.0186137 \\
\hline & 0.050 & -0.0104381 & -0.0102639 & -0.0102393 & -0.0096144 & -0.0094212 & -0.0094015 \\
\hline & 0.075 & -0.0035370 & -0.0024817 & -0.0026443 & -0.0031607 & -0.0020415 & -0.0022307 \\
\hline $5 p$ & 0.025 & -0.0098161 & -0.0098090 & -0.0098079 & -0.0080910 & -0.0080822 & -0.0080816 \\
\hline $5 d$ & 0.025 & -0.0095365 & -0.0095178 & -0.0095114 & -0.0085661 & -0.0085444 & -0.0085415 \\
\hline $5 f$ & 0.025 & -0.0093215 & -0.0092918 & -0.0092825 & -0.0087035 & -0.0086705 & -0.0086619 \\
\hline $5 \mathrm{~g}$ & 0.025 & -0.0090833 & -0.0090534 & -0.0090330 & -0.0086680 & -0.0086347 & -0.0086150 \\
\hline $6 p$ & 0.025 & -0.0043727 & -0.0043567 & -0.0043583 & -0.0035035 & -0.0034850 & -0.0034876 \\
\hline $6 d$ & 0.025 & -0.0042067 & -0.0041607 & -0.0041650 & -0.0037257 & -0.0036756 & -0.0036813 \\
\hline $6 f$ & 0.025 & -0.0040586 & -0.0039745 & -0.0039803 & -0.0037592 & -0.0036699 & -0.0036774 \\
\hline $6 \mathrm{~g}$ & 0.025 & -0.0038781 & -0.0037582 & -0.0037611 & -0.0036832 & -0.0035576 & -0.0035623 \\
\hline
\end{tabular}

Table 2. Energy eigenvalues in atomic units $(\hbar=M=1)$ for $\lambda=20$ and $\kappa=4$.

\begin{tabular}{ccccc}
\hline States & $\alpha$ & Present & {$[40](t=\mathbf{1} / \mathbf{3})$} & $\begin{array}{c}\text { Mathematica } \\
\text { (Lucha } \text { et } \text { al. })\end{array}$ \\
\hline $2 \mathrm{p}$ & 0.025 & -0.077115 & -0.077114 & -0.077077 \\
& 0.050 & -0.308459 & -0.308459 & -0.308308 \\
& 0.075 & -0.694031 & -0.694032 & -0.693691 \\
& 0.100 & -1.233834 & -1.233830 & -1.233221 \\
& 0.150 & -2.776128 & -2.776130 & -2.774697 \\
\hline
\end{tabular}




\section{Continued}

\begin{tabular}{|c|c|c|c|c|}
\hline \multirow[t]{5}{*}{$3 p$} & 0.025 & -0.058729 & -0.058728 & -0.058677 \\
\hline & 0.050 & -0.234914 & -0.234915 & -0.234706 \\
\hline & 0.075 & -0.528558 & -0.528558 & -0.528076 \\
\hline & 0.100 & -0.939658 & -0.939658 & -0.938771 \\
\hline & 0.150 & -2.114230 & -2.114230 & -2.112036 \\
\hline \multirow[t]{4}{*}{$3 d$} & 0.025 & -0.072002 & -0.072002 & -0.071879 \\
\hline & 0.050 & -0.288008 & -0.288009 & -0.287516 \\
\hline & 0.075 & -0.648019 & -0.648019 & -0.646910 \\
\hline & 0.100 & -1.152034 & -1.152030 & -1.150055 \\
\hline \multirow[t]{3}{*}{$4 p$} & 0.025 & -0.042843 & -0.042842 & -0.042778 \\
\hline & 0.050 & -0.171370 & -0.171370 & -0.171108 \\
\hline & 0.075 & -0.385583 & -0.385584 & -0.384968 \\
\hline \multirow[t]{3}{*}{$4 d$} & 0.025 & -0.054278 & -0.054278 & -0.054114 \\
\hline & 0.050 & -0.217113 & -0.217113 & -0.216455 \\
\hline & 0.075 & -0.488504 & -0.488504 & -0.487013 \\
\hline \multirow[t]{3}{*}{$4 f$} & 0.025 & -0.065502 & -0.065501 & -0.065227 \\
\hline & 0.050 & -0.262007 & -0.262008 & -0.260909 \\
\hline & 0.075 & -0.589516 & -0.589517 & -0.587042 \\
\hline \multirow[t]{3}{*}{$5 p$} & 0.025 & -0.029456 & -0.029456 & -0.029382 \\
\hline & 0.050 & -0.117826 & -0.117826 & -0.117527 \\
\hline & 0.075 & -0.265109 & -0.265109 & -0.264427 \\
\hline \multirow[t]{3}{*}{$5 d$} & 0.025 & -0.039054 & -0.039054 & -0.038854 \\
\hline & 0.050 & -0.156217 & -0.156217 & -0.155417 \\
\hline & 0.075 & -0.351488 & -0.351488 & -0.349683 \\
\hline \multirow[t]{3}{*}{$5 f$} & 0.025 & -0.048655 & -0.048654 & -0.048301 \\
\hline & 0.050 & -0.194619 & -0.194619 & -0.193205 \\
\hline & 0.075 & -0.437892 & -0.437892 & -0.434709 \\
\hline \multirow[t]{3}{*}{$5 \mathrm{~g}$} & 0.025 & -0.058294 & -0.058292 & -0.057780 \\
\hline & 0.050 & -0.233171 & -0.233171 & -0.231122 \\
\hline & 0.075 & -0.524634 & -0.524634 & -0.520024 \\
\hline \multirow[t]{2}{*}{$6 p$} & 0.025 & -0.018570 & -0.018570 & -0.018488 \\
\hline & 0.050 & -0.074282 & -0.074282 & -0.073950 \\
\hline \multirow[t]{2}{*}{$6 d$} & 0.025 & -0.026330 & -0.026330 & -0.026100 \\
\hline & 0.050 & -0.105321 & -0.105321 & -0.104400 \\
\hline \multirow[t]{2}{*}{$6 f$} & 0.025 & -0.034307 & -0.034307 & -0.033885 \\
\hline & 0.050 & -0.137230 & -0.137230 & -0.135539 \\
\hline \multirow[t]{2}{*}{$6 \mathrm{~g}$} & 0.025 & -0.042470 & -0.042470 & -0.041831 \\
\hline & 0.050 & -0.169881 & -0.169882 & -0.167327 \\
\hline
\end{tabular}




\subsection{The Pöschl-Teller Polynomial Potential}

As another interesting special case of the class of potentials under consideration, we consider the potential of the form

$$
V(r)=\sum_{j=1}^{K} B_{j} \operatorname{sech}^{2 j}(\alpha r)
$$

which we call "Pöschl-Teller polynomial potential" since it is a polynomial function in the PTP of the form $\operatorname{sech}^{2}(\alpha r)$. The PTPP can be obtained from Equation (2) by choosing $C_{-2}=C_{-1}=C_{1}=C_{2}=C_{3}=0$. It has a very interesting aspect that it can be used to model the Gaussian function $f_{G}(r)=-\exp (-r)$ by suitably choosing the values of $\alpha, K$ and the $B_{j}$ coefficients [6].

In order to show that the potential (35) can have useful applications in physics, we study the three-dimensional SE for the attractive Gaussian potential defined by

$$
V_{G}(r)=-\gamma \exp \left(-\lambda r^{2}\right)
$$

where $\gamma>0$ is the well depth and $\lambda>0$ determines its width. Note that this potential has been introduced to model nucleon-nucleon scattering [44] and has played an important role in nuclear physics. In semiconductor physics, the potential (36) has been used as a model potential to investigate the electronic structure of the quantum dots with single or more electrons [45] [46], impurity [47] and excitons [48].

The radial SE associated with the attractive Gaussian potential (36) can be written as

$$
-\frac{\lambda \hbar^{2}}{2 M} \frac{\mathrm{d}^{2} \psi(r)}{\mathrm{d} r^{2}}+\left[\frac{\ell(\ell+1) \lambda}{2 M r^{2}}-\gamma \exp \left(-r^{2}\right)\right] \psi(r)=E \psi(r)
$$

where $r=\sqrt{\lambda} r$. By using the approximation scheme (4) in Equation (37), we obtain the following differential equation:

$$
-\frac{\lambda \hbar^{2} b^{2}}{2 M} \frac{\mathrm{d}^{2} \psi(R)}{\mathrm{d} R^{2}}+\left[\frac{\ell(\ell+1) \lambda b^{2}}{2 M R^{2}}+\sum_{j=1}^{8} B_{j} \operatorname{sech}^{2 j}(\tilde{\alpha} R)\right] \psi(R)=E \psi(R)
$$

Here, $\tilde{\alpha}=\alpha / b, R=b r$ and $B_{j}=\gamma \mathcal{B}_{j}, \quad j=1, \cdots, 8$. Using the approximation scheme (1) for the centrifugal term $1 / R^{2}$, and changing variables from $R$ to $\xi=\operatorname{sech}^{2}(\tilde{\alpha} R)$, we obtain the dimensionless SE (28) with $\varepsilon=2 M E /\left(\lambda \hbar^{2} \alpha^{2}\right)$, $c_{-2}=c_{2}=0, \tilde{b}_{j}=2 M \tilde{B}_{j} /\left(\lambda \hbar^{2} \alpha^{2}\right)$.

Figure 1 shows the variation of potential as a function of the radial position (dashed curve) and the change in discrete electron energy levels $\left(E_{n, \ell}\right)$ for different values of $n$ and $\ell$ when $\lambda=1 / a_{B}^{2}$ and $\gamma=400$ Ryd. Here $a_{B}$ and Ryd are the Bohr radius and Rydberg energy, respectively. Note that $n=0,1,2, \cdots$ and $\ell=0,1,2, \cdots$ are radial and angular quantum numbers, respectively. Our results are compared with those obtained by means of the Köksal's empirical formula [41] and the numerically calculated ones by the exact Hamiltonian diagonalization on a finite basis of Coulomb sturmian functions defined by 


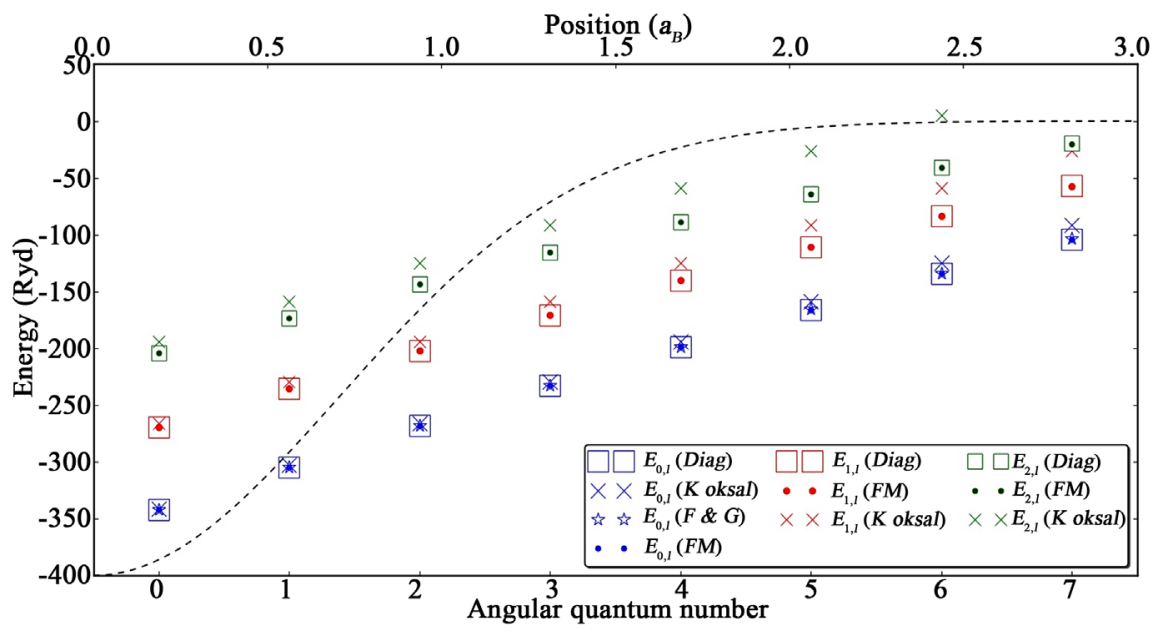

Figure 1. Bound state energy values of attractive Gaussian potential for different radial and angular quantum numbers.

$$
S_{n, \ell}^{\kappa}(r)=\mathcal{N}_{n, \ell}^{\kappa} r^{\ell+1} \mathrm{e}^{-\kappa r} L_{n-\ell-1}^{2 \ell+1}(2 \kappa r)
$$

where $L_{m}^{\alpha}(x)$ denotes the associated Laguerre polynomial and $n$ the principal quantum number. The normalization constant $\mathcal{N}_{n, l}^{\kappa}$, given by

$$
\mathcal{N}_{n, \ell}^{\kappa}=\sqrt{\frac{\kappa}{n}}(2 \kappa)^{\ell+1}\left[\frac{(n-\ell-1) !}{(n+\ell) !}\right]^{1 / 2}
$$

is obtained from the normalization condition $\int_{0}^{\infty}\left[S_{n, l}^{\kappa}(r)\right]^{*} S_{n, \ell}^{\kappa}(r) \mathrm{d} r=1$. We have chosen $N_{s}=500$ as the number of Coulomb sturmian functions and 0.75 as the value of $\kappa$. For the case of $\boldsymbol{x}=0$, we also consider results calculated by using a simple analytical expression provided by the variational method [42]. It is obvious that our results $\left(E_{n, \ell}(F M)\right)$ are much better than those obtained using the Köksal's empirical formula $\left(E_{n, \ell}(\right.$ Koksal $\left.)\right)$ [41]. They are in good agreement with the numerically calculated ones $\left(E_{n, \ell}(\right.$ Diag $\left.)\right)$. In the case of $n=0$, our results are very closer to both the numerically obtained ones, the Köksal's ones and the variational ones $\left(E_{n, \ell}(F \& G)\right)$. We appreciate that the present results appears to approach the numerical eigenvalues reasonably well even when $\pi>0$ and $\ell$ is not small, while the accuracy of the empirical formula decreases noticeably with $\ell$ and most remarkably with $n$ [41].

\section{Conclusions}

In this work, we have applied a recent approximation scheme to the centrifugal term $r^{-2}$ to solve the SE with a new class of spherically symmetric hyperbolic potentials in the framework of the FM. First, we have shown that our class of potentials can be reduced to a lot of hyperbolic or exponential potentials which are known to play a very important role in many fields of physics. Next, we have proved that the application of the FM to the family of potentials in question allows an easy determination of the bound state spectrum provided that the centrifugal potential is adequately approximated. Finally, we have calculated the 
bound state energy eigenvalues for three special cases, namely the MRP, the SPTP and the PTPP, with an application to the GPW. For each case, it is found that our results are in good agreement with those obtained by other methods for short-range potentials, i.e., small $\ell$ and $\alpha$.

One has to add that our approach can also be used to determine the approximate bound state wave functions. After substituting the calculated bound state energies to the recursion relation (26) or (30), the coefficients of the generalized series (25) can be successively determined in order to obtain the unnormalized wave functions.

\section{Acknowledgements}

Nyengeri Hippolyte would like to thank the Académie de Recherche et d'Enseignement Supérieur-Commission de la Coopération au Développement (ARES-CCD) for having provided him with a desktop and a printer which have greatly facilitated the achievement of this work.

\section{Conflicts of Interest}

The authors declare no conflicts of interest regarding the publication of this paper.

\section{References}

[1] Dong, S.H. and Garcia-Ravalo, J. (2007) Exact Solutions of the s-Wave Schrödinger Equation with Manning-Rosen Potential. Physica Scripta, 75, 307-309. https://doi.org/10.1088/0031-8949/75/3/013

[2] Qiang, W.C. and Dong, S.H. (2009) The Manning-Rosen Potential Studied by a New Approximation Scheme to the Centrifugal Term. Physica Scripta, 79, Article ID: 045004. https://doi.org/10.1088/0031-8949/79/04/045004

[3] Ikot, A.N., Antia, A.D., Akpan, I.O. and Awoga, O.A. (2013) Bound State Solutions of Schrödinger Equation with Modified Hylleraas plus Exponential Rosen Morse Potential. Revista Mexicana de Fisica, 59, 46-53.

[4] Dong, S., Garcia-Ravelo, J. and Dong, S.H. (2007) Analytical Approximations to the 1-Wave Solutions of the Schrödinger Equation with an Exponential-Type Potential. Physica Scripta, 76, 393-396. https://doi.org/10.1088/0031-8949/76/4/019

[5] Wen, F.K., Yang, Z.Y., Chong, L., Yang, W.L. and Zhang, Y.Z. (2014) Exact Polynomial Solutions of the Schrödinger Equation with Various Hyperbolic Potentials. Communications in Theoretical Physics, 61, 153-159. https://doi.org/10.1088/0253-6102/61/2/02

[6] Nyengeri, H., Simbizi, R., Girukwishaka, A., Nizigiyimana, R. and Ndenzako, E. (2018) Fröbenius Series Solutions of the Schrödinger Equation with Various Types of Symmetric Hyperbolic Potentials in One Dimension. Open Access Library Journal, 5, 1-14.

[7] Roy, A.K. (2014) Studies of Bound States Spectra of Manning-Rosen Potential. Modern Physics Letters A, 29, Article ID: 1450042. https://doi.org/10.1142/S0217732314500424

[8] Ikhdair, S.M. (2011) On the Bound-State Solutions on the Manning-Rosen Potential Including Improved Approximation to the Orbital Centrifugal Term. Physica 
Scripta, 83, Article ID: 015010. https://doi.org/10.1088/0031-8949/83/01/015010

[9] Chen, C.Y., Lu, F.L. and You, Y. (2012) Scattering States of Modified Pöschl-Teller potential in D-Dimension. Chinese Physics B, 21, Article ID: 030302. https://doi.org/10.1088/1674-1056/21/3/030302

[10] Hammed, R.H. (2012) Approximate Solution of Schrödinger Equation with Manning-Rosen Potential in Two Dimensions by Using the shifted $1 / N$ Expansion Method. Journal of Basrah Researches (Sciences), 38, 51-59.

[11] Chaudhuri, R.N. and Mondal, M. (1995) Eigenvalues of Anharmonic Oscillators and the Perturbed Coulomb Problem in N-Dimensional Space. Physical Review A, 52, Article ID: 1850. https://doi.org/10.1103/PhysRevA.52.1850

[12] Miao, C. H. and Qian, S. W. (1997) Variational Sypersymmetric WKB Approximation. Physical Review A, 56, Article ID: 2412. https://doi.org/10.1103/PhysRevA.56.2412

[13] Green, R.L. and Aldrich, C. (1976) Variational Wave Functions for a Screened Coulomb Potential. Physical Review A, 14, Article ID: 2363.

https://doi.org/10.1103/PhysRevA.14.2363

[14] Tang, A.Z. and Chan, F.T. (1987) Shifted 1/NExpansion for the Hulthen Potential. Physical Review A, 35, Article ID: 911. https://doi.org/10.1103/PhysRevA.35.911

[15] Dutt, R., Mukherji, U. and Varshni, Y.P. (1986) Shifted Large- $N$ Expansion for the Bound States of the Hellmann Potential. Physical Review A, 34, Article ID: 777. https://doi.org/10.1103/PhysRevA.34.777

[16] Nioto, M.M. (1978) Exact Wave-Function Normalization Constants for the $B_{0} \tanh z-\mathrm{U}_{0} \cosh ^{-2} z$ and Pöschl-Teller Potentials. Physical Review A, 17, 1273-1284. https://doi.org/10.1103/PhysRevA.17.1273

[17] Onate, C.A. (2015) Approximate Solutions of the Non-Relativistic Schrödinger Equation with Pöschl-Teller Potential. Chinese Journal of Physics, 53, Article ID: 060002.

[18] Ikot, A.N. (2010) Approximate Solution of the Schrödinger Equation with Rosen-Morse Potential Including the Centrifugal Term. Applied Physics Research, 2, 202-208. https://doi.org/10.5539/apr.v2n2p202

[19] Downing, C.A. (2013) On a Solution of the Schrödinger Equation with Hyperbolic Double-Well Potential. Journal of Mathematical Physics, 54, Article ID: 072101. https://doi.org/10.1063/1.4811855

[20] Hartman, R.R. (2014) Bound States in Hyperbolic Asymmetric Double-Well. Journal of Mathematical Physics, 55, Article ID: 012105. https://doi.org/10.1063/1.4861938

[21] Rosen, N. and Morse, P.M. (1932) On the Vibration of Polyatomic Molecules. Physical Review, 42, 210-217. https://doi.org/10.1103/PhysRev.42.210

[22] Zlatev, S.I. (2018) Pekeris-Type Approximation for the l-Wave in Pöschl-Teller Potential. Unpublished. https://arxiv.org/pdf/1311.5794v1.pdf

[23] Jia, C.S., Chen, T. and Cui, L.G. (2009) Approximate Analytical Solutions of the Dirac Equation with the Generalized Pöschl-Teller Potential Including the Pseudo-Centrifugal Term. Physics Letters A, 373, 1621-1626. https://doi.org/10.1016/j.physleta.2009.03.006

[24] Ourdi, R., Hassanabadi, S., Rajabi, A.A. and Hasanabadi, H. (2012) Approximate Bound State Solutions of DKP Equation for Any J State in the Presence of Woods-Saxon Potential. Communications in Theoretical Physics, 57, 15-18. https://doi.org/10.1088/0253-6102/57/1/04 
[25] Wei, G.F. and Dong, S.H. (2008) Approximately Analytical Solutions of the Manning-Rosen Potential with the Spin-Orbit Coupling Term and Spin Symmetry. Physics Letters A, 373, 49-57. https://doi.org/10.1016/j.physleta.2008.10.064

[26] Flüge, S. (1999) Practical Quantum Mechanics. Springer-Verlag, Berlin.

[27] Carrington, T. (1990) Perturbation Theory for Bending Potentials. Molecular Physics, 70, 757-766. https://doi.org/10.1080/00268979000101331

[28] Iachello, F. and Oss, S. (1993) Algebraic Model of Bending Vibrations of Complex Molecules. Chemistry Letters, 205, 285-289.

https://doi.org/10.1016/0009-2614(93)89244-C

[29] Iachello, F. and Oss, S. (1993) Vibrational Spectroscopy and Intramolecular Relaxation of Benzene. The Journal of Chemical Physics, 99, 7337-7349. https://doi.org/10.1063/1.465715

[30] Wei, G.F. and Dong, S.H. (2010) A Novel Algebraic Approach to Spin Symmetry for Dirac Equation with Scalar and Vector Second Pöschl-Teller Potential. The European Physical Journal A, 43, 185-190. https://doi.org/10.1140/epja/i2009-10901-8

[31] Hasan, Y. and Tomak, M. (2005) Nonlinear Optical Properties of a Pöschl-Teller Quantum Well. Physical Review B, 72, Article ID: 115340. https://doi.org/10.1103/PhysRevB.72.115340

[32] Manning, M.F. (1935) Energy Levels of a Symmetrical Double Minima Problem with Applications to the $\mathrm{NH}_{3}$ and $\mathrm{ND}_{3}$ Moleculs. The Journal of Chemical Physics, 3, 136-138. https://doi.org/10.1063/1.1749619

[33] Costain, C.C. and Dowling, J.M. (1960) Microwave Spectrum and Molecular Structure of Formamide. The Journal of Chemical Physics, 32, 158-165. https://doi.org/10.1063/1.1700891

[34] Fletcher, W.H. and Brown, F.B. (1963) Vibrational Spectra and the Inversion Phenomenon in Cyanamide and Deuterated Cyanamide. The Journal of Chemical Physics, 39, 2478-2490. https://doi.org/10.1063/1.1734051

[35] Hilmi, Y., Oktay, A. and Mustafa, S. (2016) Modeling of Diatomic Molecules. Molecular Physics, 114, 3134-3142. https://doi.org/10.1080/00268976.2016.1220645

[36] Manning, F.M. and Rosen, N. (1933) A Potential Function for the Vibrations of Diatomic Molecules. Physical Review, 44, 953.

[37] LeRoy, R.J. and Bernstein, R.B. (1970) Dissociation Energy and Long-Range Potential of Diatomic Molecules from Vibrational Spacings of Higher Levels. The Journal of Chemical Physics, 52, 3869-3879. https://doi.org/10.1063/1.1673585

[38] Cai, J.M., Cai, P.Y. and Inomata, A. (1986) Path-Integral Treatment of the Hulthen Potential. Physical Review A, 34, 4621-4628. https://doi.org/10.1103/PhysRevA.34.4621

[39] Bailey, D.H. (2015) A Thread-Safe Arbitrary Precision Computation Package. http://www.davidhbailey.com/dhbpapers/mpfun2015.pdf

[40] You, Y., Lu, F.L., Sun, D.S., Chen, C.Y. and Dong, S.H. (2013) Solutions of the Second Pöschl-Teller Potential Solved by an Improved Scheme to the Centrifugal Term. Few-Body Systems, 54, 2125-2132. https://doi.org/10.1007/s00601-013-0725-y

[41] Köksal, K. (2012) A Simple Analytical Expression for Bound State Energies for an Attractive Gaussian Confining Potential. Physica Scripta, 86, Article ID: 035006. https://doi.org/10.1088/0031-8949/86/03/035006

[42] Fenandez, F.M. and Garcia, J. (2013) Comment on "A Simple Analytical Expression for Bound State Energies for an Attractive Gaussian Confining Potential”. Physica 
Scripta, 87, Article ID: 027001. https://doi.org/10.1088/0031-8949/87/02/027001

[43] Lucha, W. and Schöberl, F.F. (1999) Solving the Schrödinger Equation for Bound States with Mathematica 3.0. International Journal of Modern Physics C, 10, 607-619. https://doi.org/10.1142/S0129183199000450

[44] Buck, B., Friedrich, H. and Wheatley, C. (1977) Local Potential Models for the Scattering of Complex Nuclei. Nuclear Physics A, 275, 246-268. https://doi.org/10.1016/0375-9474(77)90287-1

[45] Gomez, S. and Romero, R. (2009) Few-Electron Semiconductor Quantum Dots with Gaussian Confinement. Central European Journal of Physics, 7, 12-21.

[46] Adamowski, J., Sobkowiez, M., Szafran, B. and Bednarek, S. (2000) Electron Pair in a Gaussian Confining Potential. Physical Review B, 62, 4234-4237. https://doi.org/10.1103/PhysRevB.62.4234

[47] Wenfang and Xie (2010) Optical Properties of an Off-Center Hydrogenic Impurity in a Spherical Quantum Dot with Gaussian Potential. Superlattices and Microstructures, 48, 239-247. https://doi.org/10.1016/j.spmi.2010.04.015

[48] Hours, J., Senellart, P., Peter, E., Cavanna, A. and Bloch, J. (2005) Exciton Radiative Lifetime Controlled by the Lateral Confinement Energy in a Single Quantum Dot. Physical Review B, 71, Article ID: 161306.

https://doi.org/10.1103/PhysRevB.71.161306 